\title{
EXTRAÇÃO DE FITOBEZOAR GÁSTRICO POR VIDEOLAPAROSCOPIA: CONSIDERAÇÕES TÉCNICAS ORIGINAIS
}

\section{VIDEOLAPAROSCOPIC EXTRACTION OF GASTRIC PHYTOBEZOAR: ORIGINAL TECHNICAL ASPECTS}

\author{
Carlos Eduardo Domene, TCBC-SP1 \\ Marcos Aun ${ }^{2}$ \\ Paula Volpe ${ }^{3}$
}

\section{INTRODUÇÃO}

Designa-se como bezoar qualquer concreção de material sólido, estranho ao organismo, no interior do aparelho digestivo. Forma-se a partir da ingestão de substâncias diversas que, incompletamente digeridas ou de digestão impossível, se acumulam em segmentos variados do trato digestivo. Devido a complicações ou impossibilidade de sua mobilização por métodos conservadores ou endoscópicos, freqüentemente se impõe o tratamento cirúrgico. Realizado por laparotomia, segue-se a gastro ou enterotomia com retirada do bezoar, ou sua mobilização para segmentos distais do tubo digestivo, sem abertura visceral.

Houve apenas duas publicações do uso da laparoscopia com gastrotomia para retirada de bezoares gástricos, onde o método para mobilização e retirada do espécime pode determinar complicações ${ }^{1,2}$. Descrevemos um caso onde a conduta tomada, simples e facilmente exequiível, possivelmente demanda menos tempo e tem menor risco de complicações infecciosas.

\section{RELATO DE CASO}

Paciente masculino, de 47 anos de idade, com antecedentes de ter sido submetido à gastrectomia a Billroth I com vagotomia troncular por úlcera duodenal há 24 anos. Internou-se com dificuldade de ingerir alimentos, com náuseas e vômitos alimentares. $\mathrm{O}$ exame físico revelou leve distensão abdominal e dor à palpação profunda, sem tumor palpável, timpânico à percussão. $\mathrm{O}$ exame radiológico contrastado mostrou estômago aperistáltico com grande tumoração de contornos regulares em seu interior, esvaziando-se lentamente (Figura 1). A endoscopia digestiva alta apresentou câmara gástrica ocupada por grande bezoar formado por restos alimentares (Figura 2); o mesmo exame realizado um ano antes mostrara-se normal. A tentativa de retirada endoscópica do bezoar mostrou-se infrutífera. Optou-se pela via videolaparoscópica para a sua remoção.

Foram colocados quatro trocartes e desfeitas as aderências do fígado com o estômago operado, expondo-se a parede gástrica anterior. Um saco plástico retangular de

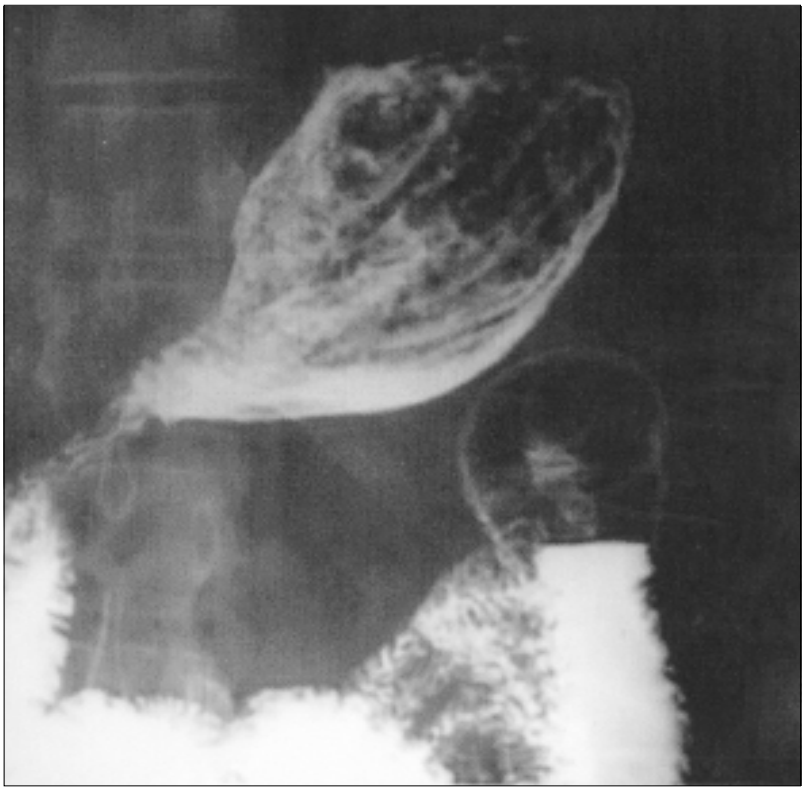

Figura 1. Radiografia contrastada de estômago revelando enorme falha de enchimento em seu interior, que pode tratar-se de bezoar.

1. Professor Livre-Docente de Cirurgia do Aparelho Digestivo pela Faculdade de Medicina da Universidade de São Paulo, São Paulo, Brasil. Diretor da Clínica Cirúrgica do Aparelho Digestivo, São Paulo, Brasil

2. Médico estagiário

3. Mestre em Cirurgia pela Faculdade de Medicina da Universidade de São Paulo

Recebido em 05/10/2001

Aceito para publicação em 12/03/2002

Trabalho realizado na Clínica Cirúrgica do Aparelho Digestivo e Hospital 9 de Julho, São Paulo-SP. 


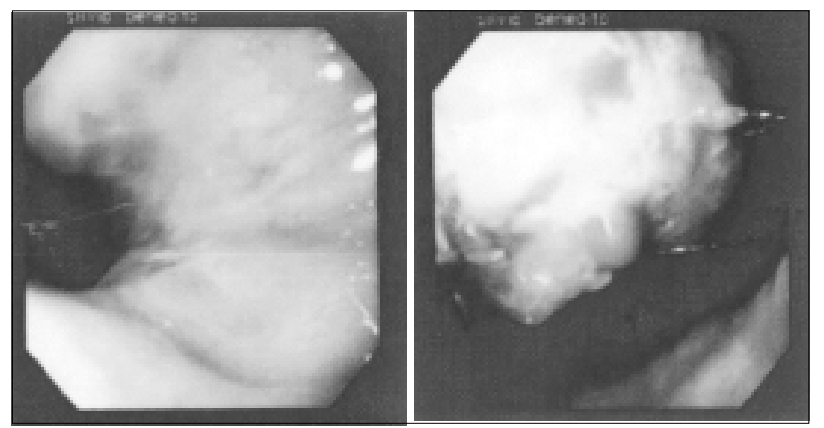

Figura 2. Esofagogastroduodenoscopia mostrando grande tumoração localizada no estômago, correspondendo a fitobezoar.

$100 \mathrm{ml}$ de capacidade foi colocado no hipocôndrio esquerdo, ao lado do estômago exposto. Foi realizada gastrotomia longitudinal com bisturi ultra-sônico e imediata aspiração do conteúdo gástrico líquido. O remanescente sólido, correspondente ao fitobezoar, foi fragmentado com pinça de babcock, sendo suas partes cuidadosamente introduzidas no saco plástico previamente colocado ao lado da gastrotomia, evitando-se com isso derramamento de conteúdo gástrico para a cavidade abdominal. Após a completa remoção do bezoar, foi realizada revisão da cavidade gástrica em busca de outros remanescentes sólidos. O saco plástico com os restos do bezoar foi fechado e colocado no hipocôndrio esquerdo. A gastrotomia foi suturada em dois planos de sutura contínua com vicryl 2-0 (mucosa e seromuscular) e a região próxima ao estômago lavada com soro fisiológico. O saco plástico foi exteriorizado pela incisão de hipocôndrio esquerdo, após a retirada do trocarte, e os fragmentos retirados com pinça convencional com extremidades rombas em forma de pá, progressivamente, sem necessidade de ampliar-se a incisão. Não foi feita drenagem da cavidade abdominal. $\mathrm{O}$ procedimento demorou 65 minutos.

O paciente recebeu alimentação líquida no primeiro dia de pós-operatório e alta no dia seguinte. Evoluiu sem qualquer intercorrência, orientado posteriormente para cuidados alimentares, medicação procinética e revisões endoscópicas periódicas. Endoscopia digestiva alta realizada um ano após a operação revelou coto gástrico sem resíduos alimentares.

\section{DISCUSSÂO}

Os métodos atualmente aceitos para o tratamento dos bezoares gástricos incluem ${ }^{3}$ : observação, dissolução enzimática, fragmentação endoscópica, laparotomia e gastrotomia. O tratamento de escolha é conservador, utilizando-se enzimas e métodos mecânicos para sua retirada. A endoscopia pode ser utilizada para quebrar-se fitobezoares pequenos e médios, retirando-se alguns segmentos e outros sendo eliminados após a fragmentação; pode-se também injetar soluções com enzimas através de cateteres endoscopicamente posicionados ${ }^{1}$. Nos bezoares muito volumosos, ou quando há complicações, o tratamento é cirúrgico. No tratamento cirúrgico de grandes bezoares gástricos realiza-se gastrotomia anterior, retirada do bezoar e sutura. Os procedimentos cirúrgicos realizados por laparotomia seguem-se de um número significativo de complicações infecciosas e incisionais ${ }^{3}$.

A videolaparoscopia vem sendo utilizada no tratamento de praticamente todas as afecções do aparelho digestivo, principalmente onde não há grandes ressecções ou o campo operatório é limitado; nessas situações, tem apresentado vantagens em relação à laparotomia com menor morbidade, mais rápida recuperação e bom resultado estético. A retirada de bezoares, ou sua mobilização distal, possivelmente se incluem dentre esses procedimentos; portanto, mais recentemente, a via videolaparoscópica pode trazer sua contribuição para o tratamento dessa doença. Descreveram-se três métodos:

1. método misto, endoscópico-laparoscópico ${ }^{4}$;

2. laparoscopia para fragmentação e mobilização distal, sem enterotomia

3. laparoscopia e gastrotomia, com retirada do bezoar por incisão suprapúbica ${ }^{1}$ ou incisão umbilical ${ }^{2}$.

Houve apenas duas publicações do uso da laparoscopia com gastrotomia para retirada de bezoares gástricos, onde o método para mobilização e retirada do espécime pode determinar complicações ${ }^{1,2}$. A técnica utilizada, em ambos os casos, foi bastante semelhante, utilizando-se três ou quatro trocartes, sendo executada gastrotomia anterior, retirada do bezoar, sua posterior colocação em saco plástico e subseqüente retirada da cavidade abdominal. No entanto, a cirurgia laparoscópica demanda utilização de recursos e cria situações onde detalhes fazem a grande diferença; em ambos os casos ocorreram possibilidades de grandes contaminações ou risco de complicações intra-operatórias que poderiam determinar conversão para laparotomia.

Descrevemos um caso em que a conduta tomada, simples e facilmente exeqüível, possivelmente demanda menos tempo e tem menor risco de complicações infecciosas.

A colocação de trocarte para óptica em situação paramediana esquerda tem o sentido de diminuir os riscos de hérnias incisionais, além de possibilitar melhor visualização do estômago. Os trocartes em hipocôndrios permitem manipulação adequada, bem como a realização de sutura intracavitária. Houve necessidade de um quarto trocarte, em posição subxifóidea, para exposição do fígado e liberação das aderências com o estômago; esse trocarte adicional foi ainda útil na execução da gastrotomia anterior, possibilitando aspiração imediata e simultânea do conteúdo gástrico líquido durante a abertura gástrica, evitando qualquer derramamento para a cavidade abdominal, além de também possibilitar melhor exposição do interior do estômago ao colocar-se o afastador em posição intragástrica.

O saco plástico, de baixo custo, já houvera sido previamente introduzido na cavidade, o que permitiu, logo após a gastrotomia realizada, passar-se à colocação direta dos fragmentos em seu interior, sem, novamente, haver perdas para o restante da cavidade abdo- 
minal. Isso impediu qualquer contaminação mais importante do abdome, além de minimizar o risco de perdas de fragmentos.

O tempo seguinte foi de fechamento do saco plástico e sutura da gastrotomia. Somente então passou-se à retirada do saco plástico através de uma das incisões, que não necessitou de ampliação devido à retirada dos fragmentos com pinça de bordos rombos, e o saco plástico ser de boa espessura e resistente. A ausência de contaminação e adequação da sutura gástrica permitiram não ser realizada a drenagem da cavidade abdominal.
Acreditamos que possa ser essa uma tática adequada para realização do procedimento proposto, onde os tempos rigidamente seguidos permitiu otimização da operação, baixa ou nenhuma contaminação, e curto tempo operatório.

A via videolaparoscópica parece, portanto, um acesso adequado para o tratamento cirúrgico dos bezoares gastrointestinais, com ou sem obstrução, podendo ser indicados para início do procedimento, convertendo-se para laparotomia se situações intra-operatórias assim o requererem.

\begin{abstract}
Videolaparoscopic surgery has been used for treatment of almost all surgical abdominal diseases, mainly where there are no large ressections, or operative field is limited. In these situations, laparoscopic surgery has the advantages of less morbidity, quick recovery and good cosmetic results. Bezoars removal, or its mobilization, is probably included in these possible proceedings. Three non-laparotomic procedures were described: 1. endoscopic-laparoscopic; 2. videolaparoscopy and mobilization of intestinal bezoar to the cecum; 3. laparoscopy and gastrotomy for bezoar removal, through suprapubic incision or the umbilical punction. There have been only two publications describing the videolaparoscopic method for bezoar removal, and the methods applied can be complications or morbidity related. We describe one case where the applied technique is simple and easy to perform, time saving and probably less complications-related. This technique, with four trocars, utilized a plastic bag besides the stomach to be opened, followed by gastrotomy, bezoar removal and immediate introduction in the plastic bag, suture of gastrotomy and removal through the left subcostal trocar. This technique was feasible and easy to perform, with short operative time, and there were no intra or post-operative complications; the patient was discharged in the second post-operative day, and is without further problems after one year followup. We believe that this could be an adequate technique to perform laparoscopic gastric bezoar removal, and the rigid sequence of operative events allows a quick procedure, with minimal contamination. The videolaparoscopy seems to be an adequate access to surgical treatment of gastro-intestinal bezoars, with or without obstruction, and should be the ellected the procedure of choice to begin the surgical treatment, with convertion to laparotomy in case of any intra-operative adversity.
\end{abstract}

Key Words: Phytobezoar; Surgical treatment; Videolaparoscopy; Laparoscopy.

\title{
REFERÊNCIAS
}

1. Nirasawa $\mathrm{Y}$, Mori $\mathrm{T}$, Ito $\mathrm{Y}$ et al. Laparoscopic removal of a large gastric trichobezoar. J Ped Surg 1998, 33:663-5

2. Yao CC, Wong HH, Chen CC et al. Laparoscopic removal of large gastric phytobezoar. Surg Laparosc Endosc Percutan Techn 2000, 10:243-5

3. Ishioka S, Sakai P, Regis OE et al. Obstructive syndrome due to a bezoar in a BII gastric stump. Endoscopic treatment. Endoscopy 1983, 15:44-6

4. Filipi CJ, Perdikis G, Hinder RA et al. An intraluminal surgical approach to the mamagement of gastric bezoars. Surg Endosc 1995, 9:831-3
5. Robles R, Lujan JA, Parrilla P et al. Laparoscopic surgery in the treatment of small bowell obstruction by bezoar. Br J Surg 1995, 82:520

Endereço para correspondência:

Carlos Eduardo Domene

Av. Arnolfo de Azevedo, 201

01236-030 — São Paulo-SP

Email:domene@netway.com.br 